\title{
MATRIX SEMIGROUPS
}

\author{
MOHAN S. PUTCHA
}

\begin{abstract}
Let $S$ be a semigroup of matrices over a field such that a power of each element lies in a subgroup (i.e., each element has a Drazin inverse within the semigroup). The main theorem of this paper is that there exist ideals $I_{0}, \ldots, I_{t}$ of $S$ such that $I_{0} \subseteq \cdots \subseteq I_{t}=S, I_{0}$ is completely simple, and each Rees factor semigroup $I_{k} / I_{k-1}, k=1, \ldots, t$, is either completely 0 -simple or else a nilpotent semigroup. The basic technique is to study the Zariski closure of $S$, which is a linear algebraic semigroup.
\end{abstract}

Let $S$ be a semigroup. We say that $S$ is strongly $\pi$-regular (s $\pi \mathrm{r}$ ) if a power of each element of $S$ lies in a subgroup of $S$. These semigroups were first studied by Azumaya [1], Drazin [4] and Munn [6]. Recently these semigroups have also been called "quasiperiodic" and "group bound." We let $\mathcal{G}, \mathcal{R}, \mathcal{L}, \mathcal{D}, \mathcal{H}$ denote the usual Green's relations on $S$ (see [3]). It is well known that $\mathcal{G}=\mathscr{D}$ for s $\pi \mathrm{r}$ semigroups. A G-class $J$ of $S$ is regular if it contains an idempotent. We let $E(S)$ denote the set of all idempotents of $S$. If $a, b \in S$, then $a \mid b$ (a divides $b$ ) if $b \in S^{1} a S^{1}$.

Throughout this paper $Z^{+}$will denote the set of all positive integers and $K$ will denote an algebraically closed field. If $n \in Z^{+}$then $\mathfrak{R}_{n}(K)$ will denote the algebra of all $n \times n$ matrices over $K$. If $a \in \Re_{n}(K)$ then we let $\rho(a)$ denote the rank of $a$. If $e, a \in \Re_{n}(K), e^{2}=e$, then we let

$$
\operatorname{det}_{e}(a)=\operatorname{det}(e a e+1-e),
$$

where 'det' denotes the determinant and 1 is the identity matrix. It is easily seen that

$$
\operatorname{det}_{e}(a) \neq 0 \text { if and only if eae } \mathcal{H} e .
$$

We consider $\Re_{n}(K)$ with its Zariski topology (see [9]). A subset of $\Re_{n}(K)$ is closed if it is the zero set of a collection of polynomials in $n^{2}$ variables. By the Hilbert Basis Theorem, closed sets satisfy the descending chain condition. Hence, every nonempty family of closed sets has at least one minimal element with respect to inclusion. If $X \subseteq \Re_{n}(K)$, then we let $\bar{X}$ denote its closure. A closed (multiplicative) subsemigroup of $\Re_{n}(K)$ will be called an algebraic semigroup. Clearly the closure of any subsemigroup of $\mathfrak{T}_{n}(K)$ is an algebraic semigroup. It has been pointed out by W. E. Clark that every algebraic semigroup is s $\pi \mathrm{r}$ (see [7, Corollary 1.4]). If $S$ is any s $\pi$ r subsemigroup of $\Re_{n}(K)$, then for all $a \in S, a^{n}$ lies in a subgroup of $S$.

\footnotetext{
Received by the editors July 26, 1982.

1980 Mathematics Subject Classification. Primary 20M10; Secondary 20 M30.

Key words and phrases. Matrix semigroup, algebraic semigroup, nil, nilpotent, chain conditions.

${ }^{1}$ This research was partially supported by NSF Grant MCS 8025597.
} 
LEMMA 1. Let $S$ be an algebraic semigroup and I a closed ideal of $S$ such that $S / I$ is nil. Then $S / I$ is nilpotent.

Proof. Suppose $S / I$ is not nilpotent. Let $\mathbb{Q}=\{T \mid T$ is a closed subsemigroup of $S, T^{m} \nsubseteq I$ for all $\left.m \in Z^{+}\right\}$. Then $S \in \mathbb{Q}$. Let $T$ be a minimal element of $Q$ with respect to inclusion. Then $\overline{T^{2}} \in \mathcal{Q}$. So $\overline{T^{2}}=T$. Thus

$$
\overline{T^{i}}=T \text { for all } i \in Z^{+} .
$$

Let $a \in T$. Suppose $\overline{T a}=T$. Then $T=\overline{T a^{i}}$ for all $i \in Z^{+}$. But $a^{i} \in I$ for some $i \in Z^{+}$. So $T \subseteq I$, a contradiction. Hence $\overline{T a} \neq T$. Thus

$$
\overline{T a} \notin Q \quad \text { for all } a \in T .
$$

Let $\mathscr{G}=\{M \mid M$ is a closed ideal of $T, T M \not I\}$. By (3), $T \in \mathscr{B}$. Let $M$ be a minimal element of $\mathscr{B}$ with respect to inclusion. If $T^{3} M \subseteq I$, then by (3), $\overline{T M}$ $=\overline{T^{3} M} \subseteq \bar{I}=I$, a contradiction. Hence $T^{3} M \nsubseteq I$. So for some $a \in T, T T a M \subseteq I$. Let $M_{0}=\overline{T a M}$. Then $M_{0}$ is a closed ideal of $T, M_{0} \subseteq M$ and $T M_{0} \not I$. So $M_{0} \in \Re$.

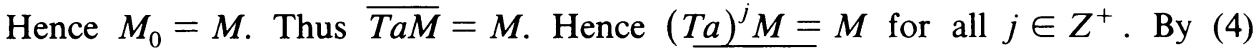
$(\overline{T a})^{k} \subseteq I$ for some $k \in Z^{+}$. So $T M \subseteq M=\overline{(T a)^{k} M} \subseteq I$, a contradiction. Hence $S / I$ is nilpotent.

REMARK 2. It is well known that if $S$ is any nil subsemigroup of $\Re_{n}(K)$, then it is nilpotent (see [5, Proposition 17.9]). If $S$ is nil, then so is $\bar{S}$. Thus Lemma 1 is a generalization of this result.

LEMMa 3. Let $S$ be an $s \pi r$ semigroup. Then:

(1) If $S_{1}$ is an $s \pi r$ subsemigroup of $S$, if $e \in E\left(S_{1}\right)$ and if $H, H_{1}$ are the $\mathcal{H}$-classes of $e$ in $S$ and $S_{1}$, respectively, then $H \cap S_{1}=H_{1}$.

(2) Any ideal of $S$ is an $s \pi r$ semigroup.

Proof. (1) Clearly $H_{1} \subseteq H \cap S_{1}$. Let $a \in S_{1} \cap H$. Then $a^{m} \mathcal{H} f$ in $S_{1}$ for some $f \in E\left(S_{1}\right)$. So $f \in a S_{1} \cap S_{1} a$. In $S$, e $\mathcal{H} a \mathcal{H} a^{m} \mathcal{H} f$. So $e=f$. Thus $f a=a f=a$. It follows that $a \mathcal{H} f=e$ in $S_{1}$. So $a \in H_{1}$.

(2) Let $I$ be an ideal of $S, a \in I$. Then $a^{m} \mathcal{H} e$ in $S$ for some $e \in E(S)$. Let $H$ denote the $\mathcal{H}$-class of $e$ in $S$. Then $a^{m} \in H \cap I$ and $H \cap I$ is an ideal of $H$. So $H \cap I=H$ and $a^{m} H e$ in $I$.

LemMA 4. Let $a, b, e \in \mathfrak{T}_{n}(K), a b=e=e^{2}, \rho(a)=\rho(e)$. Then $e a=a$.

Proof. There exist invertible matrices $c, d \in \mathfrak{N}_{n}(K)$ such that $a=c e d$. So $c e d b=e$. Hence $e d b=c^{-1} e$. So $e c^{-1} e=c^{-1} e$. Thus $e p\left(c^{-1}\right) e=p\left(c^{-1}\right) e$ for any polynomial $p(\lambda)$ over $K$. But $c$ itself is a polynomial in $c^{-1}$. So $c e=e c e$. Hence $e a=$ eced $=$ ced $=a$.

THEOREM 5. Let $S$ be an $s \pi r$ subsemigroup of $\Re_{n}(K)$. Let $I$ be an ideal of $S$ such that $S / I$ is nil. Then $S / I$ is nilpotent.

Proof. Suppose the theorem is false. Let $\mathbb{Q}=\left\{S_{1} \mid S_{1}\right.$ is a closed subsemigroup of $\Re_{n}(K)$, there exists an s $\pi \mathrm{r}$ subsemigroup $S_{2}$ of $S_{1}$ and an ideal $U$ of $S_{2}$ such that 
$S_{2} / U$ is nil but not nilpotent $\}$. Then $\Re_{n}(K) \in \mathbb{Q}$. Let $S_{1}$ be a minimal element of $\mathscr{Q}$ with respect to inclusion. There exists an s $\pi \mathrm{r}$ subsemigroup $T$ of $S_{1}$ and an ideal $V$ of $T$ such that $T / V$ is nil but not nilpotent. So $E(T) \subseteq V$. Thus $W=T E(T) T \subseteq V$. By [7, Theorem 1.7] $T$ has only finitely many regular $G$-classes. So there exist $e_{1}, \ldots, e_{m} \in E(T)$ such that $W=\left(T e_{1} T\right) \cup \cdots \cup\left(T e_{m} T\right)$. Since $T$ is s $\pi \mathrm{r}, T / W$ is nil. Since $W \subseteq V$, it suffices to show that $T / W$ is nilpotent. Clearly $\bar{W}$ is an ideal of $\bar{T}$ and $\bar{T} / \bar{W}$ is nil. By Lemma $1, \bar{T} / \bar{W}$ is nilpotent. So for some $\alpha \in Z^{+}$, $T^{\alpha} \subseteq T \cap \bar{W}$. Now $T \cap \bar{W}$, being an ideal of $T$, is s $\pi \mathrm{r}$ by Lemma 3. Clearly $W$ is an ideal of $T \cap \bar{W}$. If $\bar{W} \notin \mathcal{Q}$, then $(T \cap \bar{W}) / W$, being nil, is also nilpotent. Since $T^{\alpha} \subseteq T \cap \bar{W}, T / W$ is nilpotent, a contradiction. Thus $\bar{W} \in \mathbb{Q}$. Since $\bar{W} \subseteq \bar{T} \subseteq S_{1}$, we see that

$$
\bar{W}=\bar{T}=S_{1} .
$$

Let $W_{i}=T e_{i} T, i=1, \ldots, m$. Then $W=W_{1} \cup \cdots \cup W_{m}$ and each $W_{i}$ is an ideal of $T$. So each $\bar{W}_{i}$ is an ideal of $\bar{T}$ and $\bar{W}_{1} \cup \cdots \cup \bar{W}_{m}=\bar{W}$. Let $T_{i}=\bar{W}_{i} \cap T$, $i=1, \ldots, m$. Then by (5), $T=T_{1} \cup \cdots \cup T_{m}$. Clearly each $T_{i}$ is an ideal of $T$ and $W_{i} \subseteq T_{i} \subseteq \bar{W}_{i}$. By Lemma 3, each $T_{i}$ is s $\pi \mathrm{r}$. Suppose there exists $\beta \in Z^{+}$such that $T_{i}^{\beta} \subseteq W, i=1, \ldots, m$. Then $T^{\beta m} \subseteq W$, a contradiction. So for some $i \in\{1, \ldots, m\}$,

$$
T_{i}^{p} \nsubseteq W \text { for all } p \in Z^{+} \text {. }
$$

Let $e=e_{i}, \rho(e)=k$. Clearly $\rho(x) \leqslant k$ for all $x \in W_{i}$. Now an $n \times n$ matrix has rank $\leqslant k$ if and only if all its minors of order $\geqslant k+1$ are zero. Thus $\rho(x) \leqslant k$ for all $x \in \bar{W}_{i}$. Since $T_{i} \subseteq \bar{W}_{i}$, we see that

$$
\rho(u) \leqslant k \text { for all } u \in T_{i} .
$$

Now $Y=\left\{u \in \bar{T} \mid \operatorname{det}_{e}(x a y)=0\right.$ for all $\left.x, y \in T_{i}\right\}$ is closed and $e \notin Y$. So $Y \neq \bar{T}$. Let $M=\left\{a \in T_{i} \mid a \nmid e\right.$ in $\left.T_{i}\right\}$. Suppose $M \neq \varnothing$. Then $M$ is an ideal of $T_{i}$ and, hence, is an $\mathrm{s} \pi \mathrm{r}$ semigroup. We claim that $M \subseteq Y$. For suppose $a \in M, a \notin Y$. Then $\operatorname{det}_{e}(x a y) \neq 0$ for some $x, y \in T_{i}$. So by (2), exaye $\mathcal{H} e$ in $\Re_{n}(K)$. By Lemma 3 , exaye $\mathcal{H C} e$ in $T_{i}$. So $a \mid e$ in $T_{i}$, a contradiction. Thus $M \subseteq Y$. So $\bar{M} \subseteq Y$ and $\bar{M} \neq \bar{T}$. Hence $\bar{M} \notin \mathbb{Q}$. So $M /(M \cap W)$, being a nil semigroup, must be nilpotent. Thus there exists $\gamma \in Z^{+}$such that

$$
M^{\gamma} \subseteq W \text { or } M=\varnothing .
$$

We claim that $T_{i} \subseteq W \cup M$. So let $a \in T_{i}, a \notin M$. Then $a \mid e$ in $T_{i}$. So $x a y=e$ for some $x, y \in T_{i}$. Let $f=$ ayex $\in E\left(T_{i}\right) \subseteq W$. Now xfay $=e$. So $\rho(a) \geqslant \rho(f)=\rho(e)$ $=k$. Thus by (7), $\rho(a)=k$. So by Lemma $4, a=f a \in W$. Hence $T_{i} \subseteq W \cup M$. Since $W$ is an ideal of $T$, we see by (8) that $T_{i}^{\gamma} \subseteq W$, contradicting (6). This proves the theorem.

COROLlaRY 6. Let $S$ be an algebraic semigroup, I an ideal of $S$. If $S / I$ is nil, then it must be nilpotent.

Corollary 7. Let $S$ be an algebraic semigroup, $I$ an ideal of $S$. Then $\bar{I} / I$ is a nilpotent semigroup.

Proof. By [8, Theorem 2.3], $\bar{I} / I$ is nil. So by Theorem $5, \bar{I} / I$ is nilpotent. 
COROllary 8. Let $S$ be an $s \pi r$ subsemigroup of $\mathfrak{T}_{n}(K)$. Let $I$ be an ideal of $S$. Then there exists $p \in Z^{+}, e_{1}, \ldots, e_{m} \in E(I)$ such that $I^{p}=I^{p+1}=S e_{1} S$ $\cup \cdots \cup S e_{m} S$.

Proof. Let $M=S E(I) S$. Then, clearly $M \subseteq I^{k}$ for all $k \in Z^{+}$. Since $I$ is $s \pi r$, $I / M$ is nil. By Theorem 5, there exists $p \in Z^{+}$such that $I^{p} \subseteq M$. So $I^{p+1} \subseteq I^{p} \subseteq M$ $\subseteq I^{p+1}$. By [7, Theorem 1.7], $S$ has only finitely many regular $G$-classes. Hence there exist $e_{1}, \ldots, e_{m} \in E(I)$ such that $M=S e_{1} S \cup \cdots \cup S e_{m} S$.

Clark [2] showed that if $S$ is an $\mathrm{s} \pi \mathrm{r}$ subsemigroup of $\mathfrak{M}_{n}(K)$, then it has a completely simple kernel consisting of elements of $S$ of minimal rank. The author [7, Theorem 1.10] has shown that $S$ has ideals $I_{1}, \ldots, I_{t}$ such that $I_{1} \subseteq \cdots \subseteq I_{t}=S, I_{1}$ is completely simple, and each Rees factor semigroup $I_{k} / I_{k-1}$ is either completely 0 -simple or nil. By Theorem 5, the nil factors are all nilpotent.

THEOREM 9. Let $S$ be an $s \pi r$ subsemigroup of $\Re_{n}(K)$. Then:

(i) There exists $m \in Z^{+}, m \geqslant 2$, ideals $I_{1}, \ldots, I_{t}$ of $S$ such that $I_{1} \subseteq \cdots \subseteq I_{t}=S$. $I_{1}$ is the completely simple kernel of $S$, and for $k=2, \ldots, t$, either $I_{k}^{m} \subseteq I_{k-1}$ or $I_{k} / I_{k-1}$ is a completely 0 -simple semigroup.

(ii) $S$ does not not have a chain of more than $m^{t}$ principal right or left ideals.

(iii) $S$ does not have a chain of more than $m^{2 t}$ principal two-sided ideals.

(iv) $S$ satisfies the ascending and descending chain conditions on principal right, left and two-sided ideals.

Proof. We have already proven (i) above. So we next prove (ii). Let $q=m^{t}$. Suppose there exist $a_{1}, \ldots, a_{q+1} \in S$ such that

$$
a_{1} S^{1} \supsetneqq a_{2} S^{1} \supsetneqq \cdots \supsetneqq a_{q+1} S^{1} \text {. }
$$

Then there exist $x_{1}, \ldots, x_{q} \in S$ such that $a_{j} x_{j}=a_{j+1}, j=1, \ldots, q$. We first claim that for some $1 \leqslant \alpha<\beta<\gamma \leqslant q, x_{\alpha} \cdots x_{\beta} \Re x_{\alpha} \cdots x_{\gamma}$. We prove this by induction on $t$. Let $u_{1}=x_{1} \cdots x_{m}, u_{2}=x_{m+1} \cdots x_{2 m}, \ldots$ If each $u_{p} \in I_{t-1}, p=1, \ldots, m^{t-1}$, then we are done by induction. Suppose then that some $u_{p} \notin I_{t-1}$. If $S / I_{t-1}$ is nilpotent, then $u_{p} \in S^{m} \subseteq I_{t-1}$, a contradiction. So $S / I_{t-1}$ is completely 0 -simple. Clearly $x_{p m}, x_{p m-1}, x_{p m-1} x_{p m} \in S \backslash I_{t-1}$. Since $S / I_{t-1}$ is completely 0 -simple, it follows that $x_{p m-1} x_{p m} \Re x_{p m-1}$. Thus our claim is true. So for some $1 \leqslant \alpha<\beta<\gamma$ $\leqslant q, x_{\alpha} \cdots x_{\beta} \Re x_{\alpha} \cdots x_{\gamma}$. Thus

$$
a_{\beta+1}=a_{\alpha} x_{\alpha} \cdots x_{\beta} \Re a_{\alpha} x_{\alpha} \cdots x_{\gamma}=a_{\gamma+1} .
$$

Hence $a_{\beta+1} S^{1}=a_{\gamma+1} S^{1}$, contradicting (9). This proves (ii).

Now we prove (iii). Let $q=m^{t}, \theta=q^{2}+1$. Suppose there exist $a_{1}, \ldots, a_{\theta} \in S$ such that

$$
S^{1} a_{1} S^{1} \supsetneq \cdots \supsetneqq S^{1} a_{\theta} S^{1}
$$

There exist $x_{1}, \ldots, x_{\theta-1}, y_{1}, \ldots, y_{\theta-1} \in S^{1}$ such that $x_{j} a_{j} y_{j}=a_{j+1}, j=1, \ldots, \theta-1$. Let $x_{0}=y_{0}=1$. Let $b_{j+1}=a_{1} y_{0} \cdots y_{j}, c_{j+1}=x_{j} \cdots x_{0} a_{1}, j=0, \ldots, \theta-1$. So

$$
b_{1} S^{1} \supseteq b_{2} S^{1} \supseteq \cdots \supseteq b_{\theta} S^{1}, \quad S^{1} c_{1} \supseteq S^{1} c_{2} \supseteq \cdots \supseteq S^{1} c_{\theta} .
$$


Let $X=\left\{b_{1} S^{1}, \ldots, b_{\theta} S^{1}\right\}, Y=\left\{S^{1} c_{1}, \ldots, S^{1} c_{\theta}\right\}$. By (ii) $|X| \leqslant q,|Y| \leqslant q$. Let $W=$ $\{1, \ldots, \theta\}$. Define $\phi: W \rightarrow X \times Y$ as $\phi(j)=\left(b_{j} S^{1}, S^{1} c_{j}\right)$. Since $\theta=q^{2}+1$, there exist $\alpha, \beta \in W$ such that $\alpha \neq \beta, \phi(\alpha)=\phi(\beta)$. So $b_{\alpha} S^{1}=b_{\beta} S^{1}, S^{1} c_{\alpha}=S^{1} c_{\beta}$. Thus

$$
\begin{aligned}
S^{1} a_{\alpha} S^{1} & =S^{1} x_{\alpha} \cdots x_{0} a_{1} y_{0} \cdots y_{\alpha} S^{1}=S^{1} c_{\alpha} y_{0} \cdots y_{\alpha} S^{1}=S^{1} c_{\beta} y_{0} \cdots y_{\alpha} S^{1} \\
& =S^{1} x_{\beta} \cdots x_{0} a_{1} y_{0} \cdots y_{\alpha} S^{1}=S^{1} x_{\beta} \cdots x_{0} b_{\alpha} S^{1}=S^{1} x_{\beta} \cdots x_{0} b_{\beta} S^{1} \\
& =S^{1} x_{\beta} \cdots x_{0} a_{1} y_{0} \cdots y_{\beta} S^{1}=S^{1} a_{\beta} S^{1} .
\end{aligned}
$$

Thus $S^{1} a_{\alpha} S^{1}=S^{1} a_{\beta} S^{1}$, contradicting (10).

Clearly (iv) is a consequence of (ii) and (iii). This proves the theorem.

\section{REFERENCES}

1. G. Azumaya, Strongly $\pi$-regular rings, J. Fac. Sci. Hokkaido Univ. 13 (1954), 34-39.

2. W. E. Clark, Remarks on the kernel of a matrix semigroup, Czechoslovak Math. J. 15(90) (1965), $305-310$.

3. A. H. Clifford and G. B. Preston, The algebraic theory of semigroups, Vol. 1, Math. Surveys, no. 7. Amer. Math. Soc., Providence, R.I., 1961.

4. M. P. Drazin, Pseudo-inverses in associative rings and semigroups, Amer. Math. Monthly 7 (1958), 506-514

5. C. Faith, Algebra. II. Ring theory, Springer-Verlag, Berlin and New York, 1976.

6. W. D. Munn, Pseudo-inverses in semigroups, Proc. Cambridge Philos. Soc. 57 (1961), 247-250.

7. M. S. Putcha, On linear algebraic semigroups, Trans. Amer. Math. Soc. 259 (1980), 457-469.

8. $\ldots$ On linear algebraic semigroups. II, Trans. Amer. Math. Soc. 259 (1980), 471-491.

9. I. R. Shafarevich, Basic algebraic geometry, Springer-Verlag, Berlin and New York, 1977.

Department of Mathematics, North Carolina State University, Raleigh, North Carolina 27650 